researchers thus obtained the structures of human GPR52 in the ligand-free (apo) state and in complex with c17, a synthetic molecule that acts as an agonist (that is, it activates the receptor).

Not unexpectedly, GPR52-apo adopts the GPCR architecture that has been seen in many other structures, involving seven transmembrane domains. Surprisingly, a region of the receptor known as extracellular loop 2 (ECL2) folds into what would normally be the binding site for an endogenous ligand (the orthosteric binding site), where it acts as a lid that blocks the entrance to this site (Fig. 1). Lin et al. observed that the activity of GPR52 is significantly diminished when ECL2 is mutated or deleted, indicating that the loop is essential for signalling activity in the receptor's native environment. Meanwhile, the crystal structure of the receptor in complex with $\mathrm{c} 17$ suggests that this agonist binds to a 'side pocket' that has not been observed in previously reported structures of GPCRs. The authors therefore speculate that c17 acts allosterically - at a site remote from the orthosteric binding site - to potentiate GPR52's activity.

Remarkably, the authors were then able to form a stable complex of GPR52 with a modified $\mathrm{G}_{\mathrm{s}}$ protein in the absence of an agonist, and to obtain the structure of the complex using cryo-electron microscopy. The receptor in this complex has the structural hallmarks of previously visualized, active GPCRs captured in complex with $G$ proteins ${ }^{1}$. The arrangement of ECL2 in this active-state structure is the same as in the crystal structure of GPR52apo, implying that ECL2 acts as a 'tethered agonist' under physiological conditions to facilitate signalling pathways in the absence of an endogenous agonist - similarly to the behaviour of some other GPCRs, such as the PAR1 protease-activated receptor ${ }^{4}$.

Most GPCRs have some basal (constitutive) activity wherein they spontaneously couple to their particular $G$ proteins. The constitutive activity of GPR52 is exceptionally high $^{5}$. Indeed, Lin and colleagues find that GPR52's basal activity is so great that the receptor's ability to signal by increasing cAMP levels is only slightly augmented by the addition of $\mathrm{c} 17$.

The authors report that this high level of constitutive activity is achieved by at least two structural features that are unusual for GPCRs: the lack of a binding site for sodium ions, and the occupation of an apparent agonist-binding site by the tethered agonist in ECL2. The sodium-binding site of GPCRs is known to be important for damping constitutive activity ${ }^{6}$, and so the observation that a GPCR that lacks such a site has a high level of basal activity is not entirely surprising. By contrast, the discovery of a tethered agonist that helps to maintain GPR52 in the active state in the absence of an external agonist is truly striking. The new findings raise the intriguing possibility that, for at least some oGPCRs, the incorporation of agonists within the receptor itself obviates the need for external ligands. Indeed, several other oGPCRs that have high constitutive activities ${ }^{5}$ have been identified, along with others that don't have sodium-binding sites ${ }^{6}$.

It should be kept in mind that - as with all structural studies - Lin and colleagues' work has provided only a few snapshots of the receptor structure. Further biochemical and biophysical studies will be essential to work out the details of GPR52's dynamic behaviour under physiological conditions.

Nevertheless, the authors' high-resolution structures should aid the development of drugs that selectively target GPR52, but avoid other potential drug targets - for instance, by enabling computational studies ${ }^{7}$ in which ultra-large libraries of potential ligands are docked into the binding site revealed by the structures. Moreover, if the approaches used by Lin et al. for the structural elucidation of GPR52 are applied to other oGPCRs that have high constitutive activity ${ }^{5,6}$, they might transform our understanding of oGPCRs and accelerate their therapeutic exploitation.

Brian Krumm and Bryan L. Roth are in the Department of Pharmacology, University of North Carolina School of Medicine, Chapel Hill, North Carolina 27514, USA.

e-mail: bryan_roth@med.unc.edu

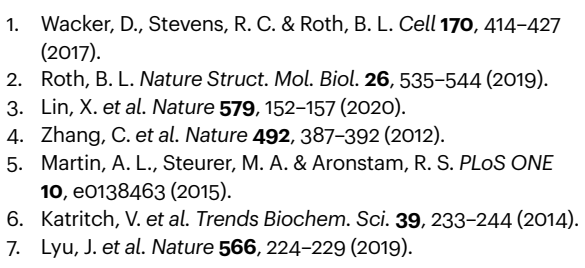

This article was published online on 19 February 2020.

\title{
Ecology
}

\section{Biodiversity theory backed by island bird data}

\begin{abstract}
Kostas A. Triantis \& Thomas J. Matthews
Analysis of a unique global data set reveals how the species diversity of birds is affected by the properties of archipelagos and offers a way to test an influential theory. Has this improved our understanding of island biodiversity patterns? See p.92
\end{abstract}

The thousands of islands in the Aegean Sea between Greece and Turkey have inspired countless myths and works of literature. This region is also where the word archipelago, which means a group of islands, has its roots. Archipelagos and their constituent islands have long been viewed as natural 'laboratories' for developing and testing theories that aim to answer key questions about biodiversity ${ }^{1-5}$. On page 92 , Valente et al. ${ }^{6}$ report an impressive analysis of birds on archipelagos worldwide that provides some of these long-awaited answers.

In the 1960s, the biologists R. H. MacArthur and E. O. Wilson proposed the theory of island biogeography ${ }^{7,8}$, which is commonly used to explain observed patterns of species richness (the number of different species) on islands. This development marked the dawning of a renaissance for biogeography (the study of species distributions over space and time) that advanced this field from a largely descriptive endeavour to a quantitative and predictive science $^{1-5}$.

The theory of island biogeography was inspired by two well-established patterns of species diversity. One pattern is that species richness increases if a greater area is sampled. The other pattern is that the species richness of an island is lower the greater the isolation of the island - the farther away the island is from a potential source of species, such as the closest mainland. The theory of island biogeography predicts that the species richness observed on an island is the result of the interplay between three fundamental processes - extinction, colonization (the dispersal and establishment of species from the continental landmass to an island) and speciation (the generation of new species) - and that these processes depend on island area and isolation. This theory has had a wide-reaching influence on researchers in fields including ecology and conservation biology, and has underpinned the emergence of subdisciplines in these fields, such as macroecology and metapopulation biology ${ }^{1-5}$.

Yet despite a multitude of studies ${ }^{3,5}$ testing the theory of island biogeography, few have sought to use molecular phylogenies to 
directly test on a global scale the dependency of extinction, colonization and speciation on island area and isolation. Valente and colleagues provide such a test. They focused on terrestrial birds, excluding migratory species, and gathered an impressive data set of 491 species across 41 archipelagos worldwide.

Building on their previous work investigating mechanisms that generate island biodiversity ${ }^{9}$, the authors applied an innovative modelling approach that combined molecular phylogenetic data with information on the spatial distribution of birds. The authors obtained genetic data from 90 species across different archipelagos, including 110 island populations not previously sampled. Valente and colleagues also sampled genetic data for the closest mainland-dwelling relatives of several of these island species. After combining their data with pre-existing data, the authors built phylogenetic trees showing the evolutionary relationships between species. Using these phylogenies, they were able to estimate colonization, extinction and speciation rates. The authors also included species known to have been driven to extinction by humans, because excluding such species impedes our understanding of natural processes and biodiversity patterns ${ }^{9,10}$.

The authors' models, which used rates estimated at the archipelago level, have high explanatory power and confirm several key predictions of the theory of island biogeography - namely, that extinction rates decline with increasing island area, colonization rates decline with increasing distance from the island to the continent, and speciation rates increase with the area and isolation of islands. The authors studied two types of speciation (Fig. 1) separately: anagenesis (in which a new species arises when an island population diverges from its ancestral species on the continent to become a different species ${ }^{3}$ ) and cladogenesis (in which an ancestral species splits into two or more different species ${ }^{3}$ ). They found that anagenesis increases with island isolation, and cladogenesis increases on larger, more isolated islands. These findings will help future studies that attempt to answer long-debated questions, such as why only certain animal and plant groups speciate extensively, and whether there are upper limits to the species richness and speciation rates in specific regions of the globe ${ }^{3}$.

Valente and colleagues have not only advanced our understanding of the laws governing species richness on islands, they have also confirmed several predictions of the theory of island biogeography. As the authors mention, the next step will be to apply their analytical framework to other island-dwelling species, particularly those, such as snails or reptiles, that have less ability to disperse than birds do. These analyses could be further
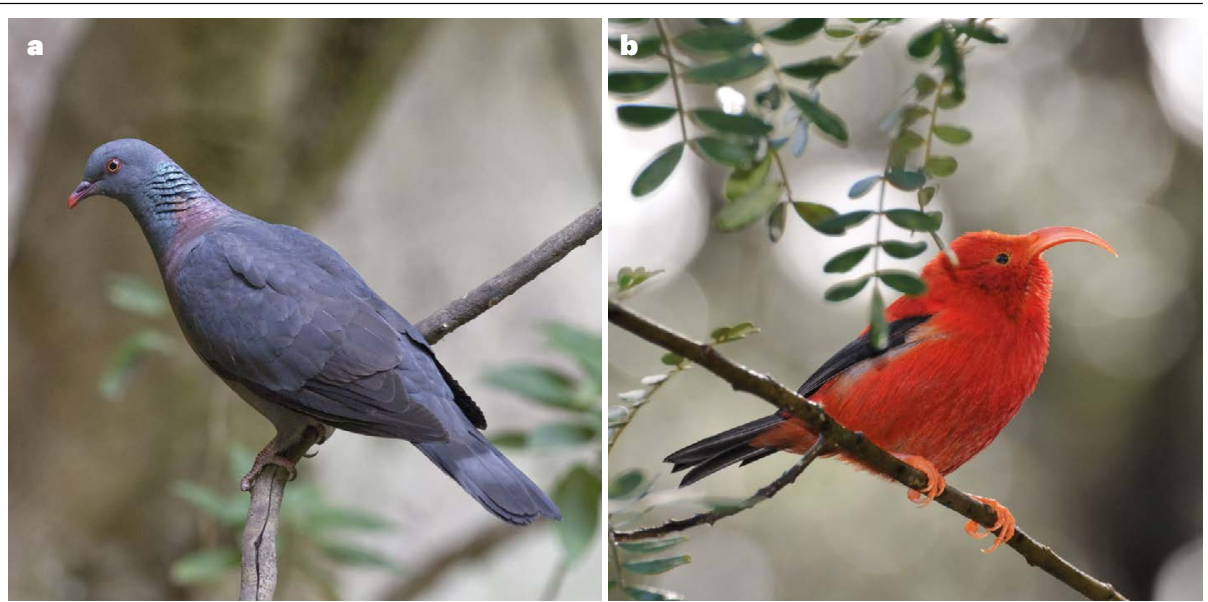

Figure 1 | Bird biodiversity. The theory of island biogeography ${ }^{7,8}$, proposed in the 1960 s, is a milestone in our understanding of how biodiversity is established and maintained. Valente et al. ${ }^{6}$ tested this theory on a global scale using data for island-dwelling birds from 41 archipelagos. Their results confirm key predictions of this theory. a, The authors report that two-thirds of the birds native to archipelagos arose from a process of species formation called anagenesis, which typically occurs on isolated islands (those far from the mainland). Anagenesis has given rise to birds such as the Bolle's pigeon (Columba bollii) of the Canary Islands. b, Another process of species formation, called cladogenesis, is most common on large, isolated islands. The authors report that of the birds they studied, a group called Hawaiian honeycreepers had the greatest number of species ( 33 in total) that arose by cladogenesis. One example of such species is Hawaii's iiwi, or scarlet honeycreeper (Drepanis coccinea).

informed by incorporating into this approach species' functional traits ${ }^{11}$, such as body size and diet.

The implications of Valente and colleagues' results extend beyond the field of island biogeography. For example, characterization of the relationship between island area and extinction rate contributes to the discussion in conservation science about how to assess the effects on biodiversity of habitat loss and fragmentation during the Anthropocene (the name proposed for the current phase of planetary history, in which human activity has a dominant influence on the environment). This is relevant to today's world, in which natural habitats are becoming increasingly isolated $^{12,13}$.

An important aspect of Valente and colleagues' study is their approach of considering an archipelago as a unit, rather than focusing on individual islands. This aligns with the idea $^{14}$ that archipelagos might be the most appropriate units in which to frame analyses of biodiversity at large spatial and temporal scales. Analysis of large spatial units in biogeography is not a new approach; however, these units generally take the form of geometric shapes, such as grid squares, that do not directly correspond to ecological boundaries (for example, those defined by vegetation type) and their associated communities. By contrast, archipelagos represent natural units. It is likely that substantial strides will be made in our understanding of island biogeography from further analyses of ecological patterns and processes undertaken at the archipelago scale, especially if geological dynamics are incorporated. To paraphrase E. O. Wilson ${ }^{15}$ : it is archipelagos that are "the logical laboratories of biogeography and evolution".

Kostas A. Triantis is in the Department of Biology, National and Kapodistrian University of Athens, Athens GR-15784, Greece. Thomas J. Matthews is at the School of Geography, Earth and Environmental Sciences, and at the Birmingham Institute of Forest Research, University of Birmingham, Birmingham B15 2TT, UK.

e-mails: ktriantis@biol.uoa.gr;

t.j.matthews@bham.ac.uk

Whittaker, R. J., Fernández-Palacios, J. M., Matthews, T. J., Borregaard, M. K. \& Triantis, K. A. Science 357, eaam8326 (2017).

2. Losos, J. B \& Ricklefs, R. E. Nature 457, 830-836 (2009).

3. Warren, B. H. et al. Ecol. Lett. 18, 200-217 (2015).

4. Gillespie, R. G. \& Clague, D. A. Encyclopedia of Islands (Univ. California Press, 2009)

5. Lomolino, M. V. \& Brown, J. H. Q. Rev. Biol. 84, 357-390 (2009).

6. Valente, L. et al. Nature 579, 92-96 (2020)

7. MacArthur, R. H. \& Wilson, E. O. Evolution 17, 373-387 (1963).

8. MacArthur, R. H. \& Wilson, E. O. The Theory of Island Biogeography (Princeton Univ. Press, 1967)

9. Valente, L. et al. Curr. Biol. 27, 1660-1666 (2017).

10. Steadman, D. W. Extinction and Biogeography of Tropical Pacific Birds (Univ. Chicago Press, 2006).

11. Pigot, A. L. et al. Nature Ecol. Evol. 4, 230-239 (2020).

12. Haddad, N. M. et al. Sci. Adv. 1, e1500052 (2015).

13. Russell, J. C. \& Kueffer, C. Annu. Rev. Environ. Resour. 44. 31-60 (2019).

14. Triantis, K. A., Economo, E. P., Guilhaumon, F. \& Ricklefs, R. E. Glob. Ecol. Biogeogr. 24, 594-605 (2015).

15. Wilson, E. O. in The Theory of Island Biogeography Revisited (eds Losos, J. B. \& Ricklefs, R. E.) 1-12 (Princeton Univ. Press, 2010)

This article was published online on 19 February 2020. 